\title{
X-RAY PHOTOELECTRON SPECTROSCOPY STUDY OF LIPID-FREE MULTILAYER SYSTEMS OF COPPER PHTHALOCYANINE TETRASULPHONATE
}

\author{
X. D. CHAI, K. TIAN, H. J. CHEN, X. Y. TANG AND T. J. LI \\ Department of Chemistry, Jilin University, Changchun, 130023 (China) \\ Z. Q. ZHU \\ Department of Physics, Henan University, Kaifeng (China) \\ D. MÖBIUS \\ Max-Planck-Institut für biophysikalische Chemie, Postfach 2841, D-3400 Göttingen (F.R.G.) \\ (Received April 25, 1989; accepted June 21, 1989)
}

Angle-dependent X-ray photoelectron spectroscopy (XPS) is an effective method to obtain structural information about a given surface of a single crystal and metal epitaxial layers. We have used this method to study the molecular stacking of a lipid-free copper phthalocyanine tetrasulphonate (CuTsPc) multilayer system. On the basis of two enhanced peaks found in the angle-dependent XPS spectrum recorded from a system of $11 \mathrm{CuTsPc}$ monolayers on gold and the mechanism of enhancement, we suggest face-to-face stacking of the CuTsPc molecules, with copper atoms stacked in the direction at $15^{\circ}$ with respect to the normal of the molecular plane. This stacking also provides a good explanation of the electrical conductivity of this multilayer system.

\section{INTRODUCTION}

In a previous communication ${ }^{1}$ we reported on a method of preparing lipid-free copper phthalocyanine tetrasulphonate (CuTsPc) multilayer systems as well as on light absorption, surface-enhanced Raman scattering and electrical conductivity measurements with such systems. The results suggested that an ordered molecular orientation must exist in these films. In this paper we study the interlayer molecular stacking in such a multilayer system by angle-dependent X-ray photoelectron spectroscopy (XPS).

Angle-dependent XPS is generally used to obtain structural information about a given surface of a single crystal. An important phenomenon here is the enhancement of the photoelectron emission along the direction of crystal axes ${ }^{2,3}$. This phenomenon is now interpreted as an electron-channelling effect caused by lattice diffraction of the photoelectrons ${ }^{4,5}$. Photoelectron emission enhancement was also found to be effective in the determination of atomic packing in epitaxially deposited metal layers by Egelhoff ${ }^{6}$. Even though no electron channels were formed in his epitaxial layers because of an insufficient number of layers, the enhancement is still produced for the forward scattering of photoelectrons by short-range order of overlayer atoms. Analysing all these characteristic features, we expected that it 
should be possible to study the packing of ordered organic molecular films containing atoms with effective soft X-ray diffraction by angle-dependent XPS. We report here on a first attempt with this method. The results are fairly satisfying and give a reasonable explanation for the electrical conductivity of this particular type of multilayer system.

\section{EXPERIMENTAL DETAILS}

In this experiment, lipid-free CuTsPc multilayer systems were prepared at the MPI für biophysikalische Chemie, Göttingen, F.R.G. by the technique described earlier ${ }^{1}$. Samples of 11 monolayers transferred onto glass plates covered with a vacuum-deposited gold layer of area $12 \times 12 \mathrm{~mm}^{2}$ were used, as well as samples of 5 monolayers transferred onto bare glass plates of the same area. Angle-dependent XPS was done with a Vg ESCALAB MK II instrument. The geometry is given schematically in Fig. 1 (angles with respect to the surface normal). The X-ray incident in the $x-y z$ space at an angle $\phi$ and the protensive line of the electron energy analyser at an angle $\theta$, in the $y z$ plane, are pointing to the centre of the sample. The angle between the incident X-ray and the electron energy analyser is fixed at $35^{\circ}$. Different angles $\phi$ were obtained by rotating the sample around the $y$ axis with fixed centre.

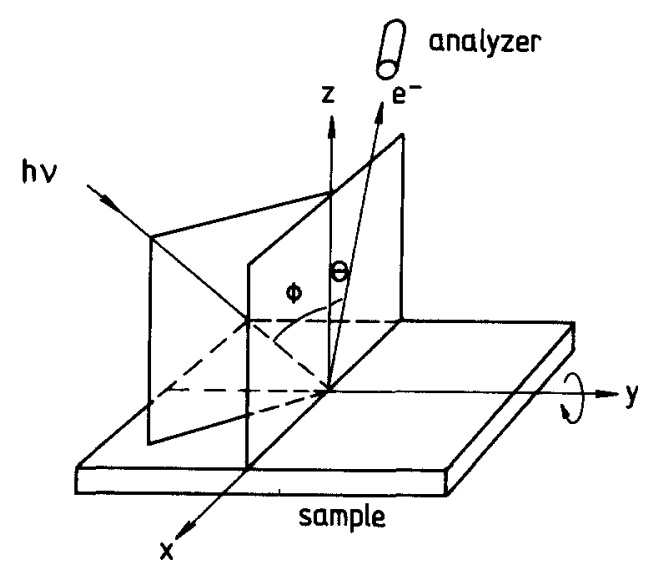

Fig. 1. Schematic diagram of angle-dependent XPS measurement.

$240 \mathrm{~W}(12 \mathrm{kV} \times 20 \mathrm{~mA})$ of total power $(\mathrm{Mg} \mathrm{K} \alpha)$ and $20 \mathrm{eV}$ electron pass energy were used. Because of the great stability of the CuTsPc multilayer system resulting from in-plane salt formation, room temperature and ultrahigh vacuum $\left(5 \times 10^{-10}\right.$ Torr $)$ conditions were selected.

The photoelectron intensities of $\mathrm{Cu} 2 \mathrm{p}_{3 / 2}$ from the CuTsPc monolayer system as well as $\mathrm{Au} 4 \mathrm{f}_{7 / 2}$ and $\mathrm{O} 1 \mathrm{~s}$ from the support were recorded at different angles $\phi\left(5^{\circ}\right.$, $15^{\circ}, 25^{\circ}, 35^{\circ}, 45^{\circ}, 50^{\circ}, 55^{\circ}$ ). To compare multilayers with disordered systems, the photoelectron intensity of $\mathrm{Cu} 2 \mathrm{p}_{3}$ from $\mathrm{CuTsPc}$ powder was measured in the same way. 


\section{RESULTS AND DISCUSSION}

Figures 2 and 3 show the $\mathrm{Cu} 2 \mathrm{p}_{3 / 2}$ angle-dependent XPS spectra of CuTsPc powder and $11 \mathrm{CuTsPc}$ monolayers respectively. In these two figures the intensity at $\phi=5^{\circ}$ was normalized to unity for comparison. Figure 2 is typical of angledependent spectra recorded on powder samples by this instrument, with a maximum intensity at about $\phi=15^{\circ}$ and a decrease in intensity with increasing $\phi$. In Figure 3 we can see two peaks at $\phi=50^{\circ}$ and $\phi=25^{\circ}$. According to the specification of the instrument, the photoelectrons are detected at $\theta=15^{\circ}$ for $\phi=50^{\circ}$. For $\phi=25^{\circ}$ the analyser is on the other side of the normal, and again $\theta \approx 15^{\circ}$. The reproducibility of these angular characteristics was good.
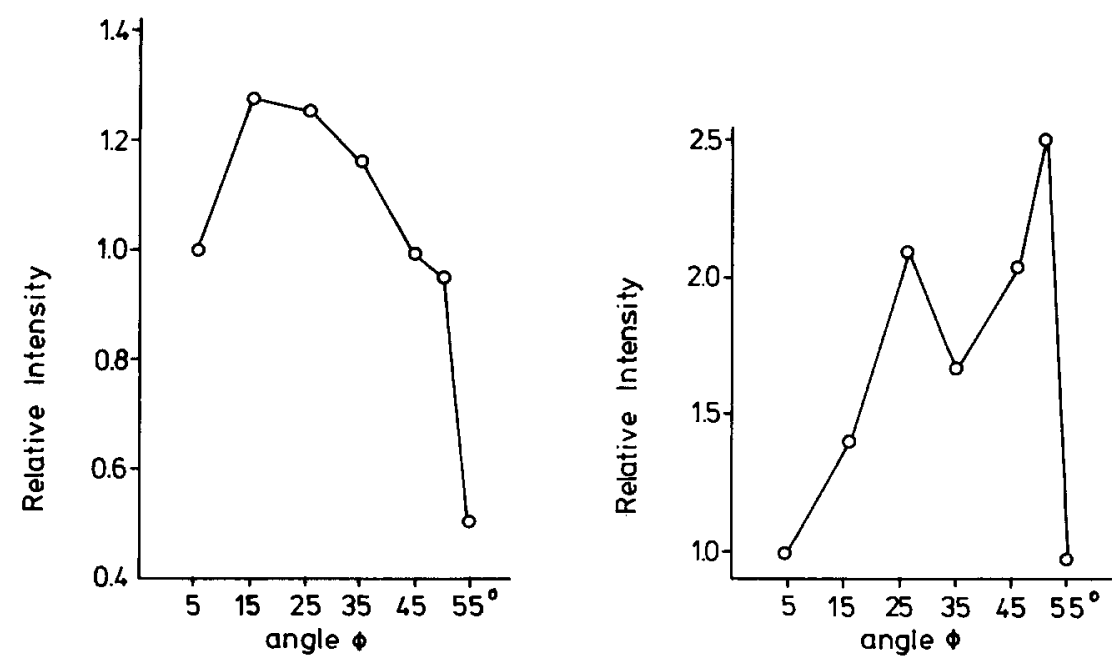

Fig. 2. $\mathrm{Cu} 2 \mathrm{p}_{3 / 2}$ angle-dependent XPS spectrum of CuTsPc powder.

Fig. 3. Cu $2 \mathrm{p}_{3 / 2}$ angle-dependent XPS spectrum of $11 \mathrm{CuTsPc}$ monolayer system.

On the basis of the enhancement mechanism mentioned above, we can conclude that an electron channel exists in this multilayer system and that the two peaks in the angular characteristics are in fact collected along the direction of this electron channel. Therefore the electron channel in this monolayer system has a direction of $15^{\circ}$ with respect to the normal of the film plane. Combining this result and the fact that the CuTsPc molecules in the monolayer, according to the measured visible absorption spectrum, exist predominantly as dimers, we suggest the diagram of the electron channel shown in Fig. 4. It should be noted that this figure represents not only the structure of the $\mathrm{Cu} 2 \mathrm{p}_{3 / 2}$ photoelectron channel but also the molecular stacking in this monolayer system.

The $\mathrm{Cu} 2 \mathrm{p}_{3 / 2}$ angle-dependent XPS spectrum recorded for 5 monolayers deposited on a smooth glass plate is similar to that shown in Fig. 3. Two peaks appear at the same two $\phi$ angles. It seems unlikely that such a strong enhancement is due to an electron-channelling effect because the maximum number of copper atoms is 10 , which is near the lowest limit of construction of an electron channel ${ }^{6}$. On the 
other hand, Egelhoff's interpretation of the enhancement effect found for metal films with a small number of monolayers (less than 4-5 monolayers) in the direction of interlayer atomic stacking as forward scattering of photoelectrons may also be valid for our systems. Thus similar conclusions about interlayer CuTsPc molecular stacking can be drawn.

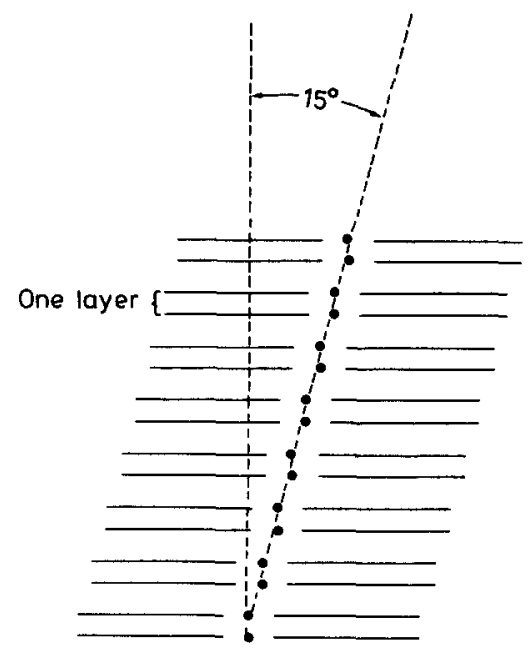

Fig. 4. Electron channel in CuTsPc multilayer systems due to shifted stacking of Pc molecules; $\bullet$, copper atom.

Further support for the structure shown in Fig. 4 is available from the analysis of angle-dependent XPS spectra of $A u 4 f_{7 / 2}$ and $O 1 s$ measured from the substrates of 11 monolayers and 5 monolayers respectively as shown in Figs. 5 and 6 . These characteristics are quite different from that measured on film-covered matrix, which is quite similar to that shown in Fig. 2. A comparison of Figs. 5 and 6 with Fig. 3
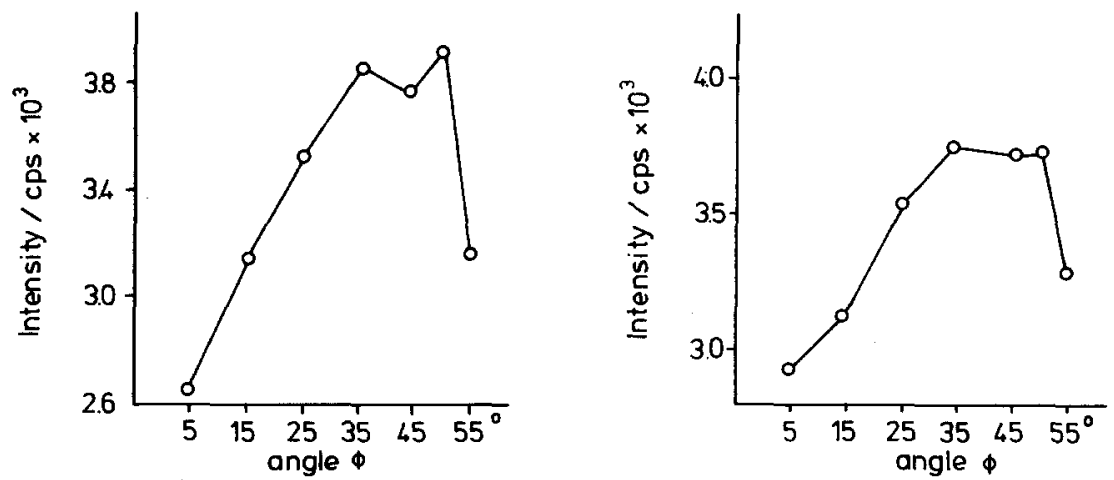

Fig. 5. Au $4 \mathrm{f}_{7 / 2}$ angle-dependent XPS spectrum recorded on gold substrate covered with $11 \mathrm{CuTsPc}$ monolayers.

Fig. 6. O 1s angle-dependent XPS spectrum recorded on glass substrate covered with 5 CuTsPc monolayers. 
suggests that they both have two enhancement peaks, one at $\phi=50^{\circ}$ as in Fig. 3 and the second at $\phi=35^{\circ}$ with $10^{\circ}$ difference from the corresponding peak at $\phi=25^{\circ}$ in Fig. 3. This means that the photoelectron coming from the substrate is also enhanced by the electron channel, but owing to the difference of photoelectron kinetic energy between $\mathrm{Au} 4 \mathrm{f}, \mathrm{O} 1 \mathrm{~s}$ and $\mathrm{Cu} 2 \mathrm{p}$, the peak positions are slightly different.

Furthermore, the molecular stacking of Fig. 4 gives a good understanding of the electrical conductivity of such monolayer systems. In this stacked structure an increase in the inter-ring overlap of the CuTsPc molecule associated with an increase in $\mathrm{Cu}-\mathrm{Cu}$ distance is observed as compared to the situation in $\alpha$-and $\beta$-type $\mathrm{CuPc}$ monocrystals ${ }^{7}$. This explains the increased electrical conductivity $\left(10^{-5} \mathrm{~S} \mathrm{~cm}^{-1}\right)$ of the stacked structure. In fact, studies on the relationship between phthalocyanine molecular stacking and electrical conductivity have demonstrated that the electrical conductivity increases with decreasing angle between the intermolecular axis and the normal of the Pc molecular plane.

\section{CONCLUSION}

In lipid-free CuTsPc multilayers the CuTsPc molecules are stacked face-toface, with the interlayer copper atoms aligned in the direction tilted $15^{\circ}$ with respect to the normal of the molecular plane.

\section{REFERENCES}

1 K. Tian, M. X. Gong, Y. B. Bai and T. J. Li, Thin Solid Films, 159 (1988) 239.

2 S. Evans and M. D. Scott, Surf. Interface Anal., 3 (1981) 269.

3 S. Evans, J. M. Adams and J. M. Thomas, Phil. Trans. R. Soc. Lond., 292 (1979) 563.

4 D. Briggs and P. M. Seah (eds.), Practical Surface Analysis, by Auger and Photoelectron Spectroscopy, Wiley, New York, 1983, p. 136.

5 C. S. Fadley and S. A. L. Bergström, Phys. Lett. A, 35 (1971) 375.

6 W. F. Egelhoff, Jr., J. Vac. Sci. Technol. A, 3 (1985) 1511.

7 E. Canadell and S. Alvarez, Inorg. Chem., 23 (1984) 573. 